\title{
Fast Algorithm for Computing Non-Isothermal Crystallization Kinetics
}

\author{
Bauke Heeg
}

Lumium, Eewal 84, Leeuwarden, the Netherlands ${ }^{*}$

\begin{abstract}
A fast algorithm is presented for computing fractional crystallized volume of an amorphous solid under non-isothermal conditions, as a function of the number of discrete time intervals, temperature history and (temperature dependent) nucleation and growth rates. The algorithm is a modification of the discrete Yinnon-Uhlmann approach to compute the standard double integral formula under quasi-steady-state conditions. Rather then re-computing the kinetics over the entire thermal history of all previous time intervals for each new interval, the crystallized fraction for a given time interval is computed by a sum of terms involving only the current and previous intervals, such that orders of magnitude increase in computational speed is obtained.
\end{abstract}

Keywords: non-isothermal crystallization, algorithm

\section{Introduction}

In many engineering problems an estimate is needed of the fractional crystallized volume $F_{V}$ of an amorphous (glassy, ceramic, polymer, semiconductor, metallic) solid under non-isothermal conditions, for a given or hypothetical nucleation frequency $I$ and growth rate $u$, and temperature as a function of time, $T(t)$. These cases can vary from simple temperature ramps such as those used in thermo-analytical methods, to a complicated thermal history. As pointed out by Yinnon and Uhlmann [1] the assumption of Arrhenian temperature dependences for either $I$ or $u$ is not often truly applicable, such that a more exact numerical method is preferable for use in thermoanalytical methods. On the other hand, for complicated temperature transients no other option exists but to use a numerical approach for computing the fraction of phase transformed. One of the main reasons for the failure of analytical approximations under highly non-isothermal conditions is that the crystal size distribution at time $t$ depends on the nucleation rate (with a temperature dependency being different from that of the growth rate) of all preceding moments

\footnotetext{
* Corresponding E-mail: bheeg@lumium.nl. Part of this work originated while at MetroLaser, Inc., Irvine CA, USA.
} 
in time in a very non-linear way. Each $(t-T)$ thermal history "trajectory" is unique, and reversing it does not lead to the same $F_{V}$. There are three exceptions: (1) the (trivial) case of a timesymmetric trajectory; (2) the case of site saturation, where crystal sizes can be computed on the basis of only the crystal growth as a function of temperature, and (3) the isokinetic hypothesis where nucleation and growth rates are assumed to have the same temperature dependence. Even in the case of these exceptions, numerical methods are preferable (or even needed) for computing $F_{V}$ under non-isothermal conditions.

Several numerical methods have been developed for the analysis of the effect of thermal history on crystallization under non-isothermal conditions, and specifically for an arbitrary thermal history. One of these approaches, the numerical computation of the quasi-steady state (QSS) suggested by Yinnon and Uhlmann [1,2] forms the starting point in the present work. The aim here is to demonstrate that significant improvements in computational speed can be achieved by re-organizing the master equation, while maintaining the same accuracy. Another main, and more general, approach is the inclusion of transient nucleation effects in the dynamics, with the rate-equation method developed by Kelton being the most widely applied [3]. While the inclusion of heating and cooling rates in the computation of crystallization dynamics is necessary in most cases [4,5], it is considered a possibility that transient effects may be implemented in the QSS in a straightforward manner, as will be briefly discussed. Thus, an in-depth comparison of different methods (including a significant amount of publications related to the theoretical aspects of non-isothermal crystallization) is postponed and the present study is limited to the improved formulation of the QSS numerical approach.

The common goal of the different methods is to enable computation of crystallization under arbitrary thermal histories. For instance, numerical approaches have been applied to estimate the thermal (single cool down) history of lunar rock [6] or earth lava [7] from the size distribution of crystallites. There are many industrial applications in which thermal history is estimated from the analysis of either $F_{V}$ or the crystal size distribution. One example is the use of the size of the gamma-prime precipitates in single crystal superalloys, obtained by post-mortem metallography, to estimate the maximum temperature of hot section gas turbine components. Another application of interest is the simulation of a glass-ceramic sensor with which an arbitrary thermal history can be, in a way, recorded and from which an approximation of the thermal history may in principle be reconstructed [8]. 


\section{Theory}

The crystallization kinetics of materials of interest are assumed to be described sufficiently accurate by the established Johnson-Mehl-Avrami-Kolmogorov (JMAK) equation within the traditional quasi-adiabatic approximation ${ }^{1}$

$$
F_{V}(t)=1-\exp \left[-g \int_{0}^{t} I\left(\int_{t^{\prime}}^{t} u d t^{\prime \prime}\right)^{m} d t^{\prime}\right],
$$

where $F_{V}(t)$ is the crystallized volume fraction as a function of time-varying temperature, $I$ and $u$ are the nucleation and growth rates, $g$ is the geometric (shape) factor (e.g., $g=4 \pi / 3$ for spherical grains), $t^{\prime}$ and $t^{\prime \prime}$ are integration variables and $m$ is the dimensionality of crystal growth. The implicit assumption in Eq. (1), also referred to as the quasi-steady-state (QSS) approximation, is that the nucleation and growth rates are a function of the instantaneous temperature, and not of the heating and/or cooling rates of the material. The ramifications of this approximation will be considered in more detail in the discussion section. Several limiting cases are known where Eq. (1) leads to incorrect results, for instance in the case of a large crystal growth anisotropy. Such exceptions are not considered at present.

The continuous equation (1) can be discretized according to Yinnon and Uhlmann as [1]

$$
F_{V}\left(t_{j}\right)=1-\exp \left[-\frac{4 \pi}{3} S_{j}\right],
$$

where $t_{j}$ is the $j^{\text {th }}$ time interval of uniform length $\Delta t$, and

$$
S_{j}=\Delta t \sum_{i=1}^{j}\left(R_{i}^{*}+\sum_{k=i}^{j} u\left(t_{k}\right) \Delta t\right)^{m} I\left(t_{i}\right),
$$

where $R_{i}^{*}$ is the critical nucleus radius. Nucleation and growth rates $I$ and $u$ can be defined as $[1,2,8]$

$$
\begin{gathered}
I(T)=\frac{K}{\eta(T)} \exp \left[-0.0205 B\left(1-\frac{T}{T_{m}}\right)^{-2}\left(\frac{T}{T_{m}}\right)^{-3}\right], \text { and } \\
u(T)=\frac{10 k T}{3 \pi \eta(T) a_{0}^{2}}\left\{1-\exp \left[-\frac{\Delta H_{m}\left(T_{m}-T\right)}{R T_{m} T}\right]\right\},
\end{gathered}
$$

where $K$ is a pre-exponential factor (assumed to be $10^{30} \mathrm{Pcm}^{-3} \mathrm{~s}^{-1}$ ), $\eta$ is the temperature dependent viscosity, $B$ is a dimensionless activation energy barrier to nucleation, expressed as a multiple of $k T, T_{m}$ is the melting point, $k$ is Boltzmann's constant, $a_{0}$ is the diameter of the rate limiting 
species (assumed to be $1 \AA$ ), $R$ is the gas constant and $\Delta H_{m}$ is the enthalpy of fusion. The viscosity, of glass forming liquids is typically modeled by the Vogel-Fulcher-Tamman (VFT) equation

$$
\eta(T)=A \exp \left[\frac{Q}{T-T_{0}}\right],
$$

where $A, Q$ and $T_{0}$ are constants for a particular liquid phase.

For significantly progressed crystallization, the contribution of the sum total of critical nuclei volume is small compared to the total volume of crystallized material, such that $R_{i}^{*}$ can be omitted from Eq. (3).

In the following sections, the improved algorithm is derived for the case of $m=3$, which corresponds to near-spherical interface-controlled growth, such as found in many glass ceramics. A similar algorithm can be derived for different values of $m$, such that the current choice of $m=$ 3 is somewhat arbitrary and based only on its purpose of demonstrating validity of the algorithm. First, Eq. (3) is written as

$$
S_{j}=\Delta t^{4} \sum_{i=1}^{j}\left(\sum_{k=i}^{j} u_{k}\right)^{3} I_{i},
$$

where $\quad I_{i}=I\left(t_{i}\right) \quad$ and $\quad u_{k}=u\left(t_{k}\right)$. For instance, $\quad S_{2}=I_{1}\left(u_{1}+u_{2}\right)^{3}+I_{2}\left(u_{2}\right)^{3}$ and $S_{3}=I_{1}\left(u_{1}+u_{2}+u_{3}\right)^{3}+I_{2}\left(u_{2}+u_{3}\right)^{3}+I_{3}\left(u_{3}\right)^{3}$, etc. It can be seen that computation of $F_{V}$ at the $j^{\text {th }}$ time interval $t_{j}$ using Eqs. (2) and (7) involves re-computing the entire thermal history that was already partially computed for the $(j-1)^{\text {th }}$ time interval. Therefore, as the crystallization is computed for increasing time interval number, the computation rapidly slows down. As will be discussed further on, depending on the average heating and cooling rates, the interval width $\Delta t$ should not be larger then a certain value to avoid inaccuracies, as a result of which the above procedure may become impractically long in the case of a large thermal history.

In order to minimize the computation time, the problem is preferably solved in a way that does not require recalculating the entire history for each incremental step. What is searched for, is the difference $\Delta S_{j}=S_{j}-S_{j-1}$, for each value of $j$. In other words, such a method requires $\Delta S_{j}$ to be calculated after interval $(j-1)$ and added to $S_{j-1}$ in order to find the fractional crystallization at time-step j, via Eq. (2). By rewriting Eq. (7) an explicit form for $\Delta S_{j}$ may be found to be a sum of 4 terms 


$$
\Delta S_{j}=S_{j A}+S_{j B}+S_{j C}+S_{j D}
$$

where

$$
\begin{aligned}
& S_{j A}=u_{j}^{3} \cdot \sum_{i=1}^{j} I_{i} \\
& S_{j B}=3 u_{j} \cdot \sum_{i=1}^{j-1} I_{i} \cdot\left(\sum_{n=i}^{j-1} u_{n}^{2}\right) \\
& S_{j C}=3 u_{j}^{2} \cdot \sum_{i=1}^{j-1} I_{i} \cdot\left(\sum_{n=i}^{j-1} u_{n}\right) \\
& S_{j D}=6 u_{j} \cdot \sum_{i=1}^{j-1} I_{i} \cdot\left(\sum_{n=i}^{j-2} u_{n} \cdot\left(\sum_{a=i+1}^{j-1} u_{a}\right)\right)
\end{aligned}
$$

In this definition, the summations still require the entire thermal history to be recomputed (i.e., all previous steps in time), however, they can be rewritten solely in terms of a single term involving all preceding intervals and another term involving just interval $j$ :

$$
\begin{aligned}
& S_{j A}=u_{j}^{3} \cdot\left[\sum_{i=1}^{j-1} I_{i}+I_{j}\right] \\
& S_{j B}=3 u_{j} \cdot\left[\frac{S_{(j-1) B}}{3 u_{j-1}}+u_{j-1}^{2} \cdot\left(\sum_{i=1}^{j-1} I_{i}\right)\right] \\
& S_{j C}=3 u_{j}^{2} \cdot\left[\frac{S_{(j-1) C}}{3 u_{j-1}^{2}}+u_{j-1} \cdot\left(\sum_{i=1}^{j-1} I_{i}\right)\right] \\
& S_{j D}=6 u_{j} \cdot\left[\frac{S_{(j-1) D}}{6 u_{j-1}}+u_{j-1} \cdot\left(u_{j-2} u_{j-3} \ldots u_{1}\right) \bullet\left(\sum_{i=1}^{j-2} I_{i} \sum_{i=1}^{j-3} I_{i} \ldots I_{1}\right)^{T}\right]
\end{aligned}
$$

where the last term contains a dot product of the vector $\left(u_{j-2} u_{j-3} \ldots u_{1}\right)$ and the transpose of the $\operatorname{vector}\left(\sum_{i=1}^{j-2} I_{i} \sum_{i=1}^{j-3} I_{i} \ldots I_{1}\right)$.

Thus a fast algorithm can be developed for calculating large thermal histories, which is referred to as Method\#2. All first terms on the right hand side (RHS) of equations (10) can be stored in memory, as well as the summations (second terms on RHS of eq. (10)) for $S_{j B}$ and $S_{j C}$ and the lower index terms of the dot product. Thus, computing $\Delta S_{j}$ involves a fixed number of relatively few operations, regardless of the interval $j$. Finally, it is noted that the computation of $F_{V}$ via Eqs. (2) and (10) is also valid for different definitions of nucleation and growth rates. The only conditions are $m=3$ and negligible $R_{i}^{*}$. 


\section{Simulation Results}

The accuracy of the fast algorithm was tested with a hypothetical glass ceramic material defined by the following constants: $A=10^{-10} \mathrm{P}, Q=39,000 \mathrm{~K}, T_{0}=0 \mathrm{~K}, B=105, T_{m}=1411 \mathrm{~K}, \Delta H_{m}=$ $280 \mathrm{~kJ} / \mathrm{mol} .^{\dagger}$ Nucleation and growth rates for these parameters are shown in Fig. 1.

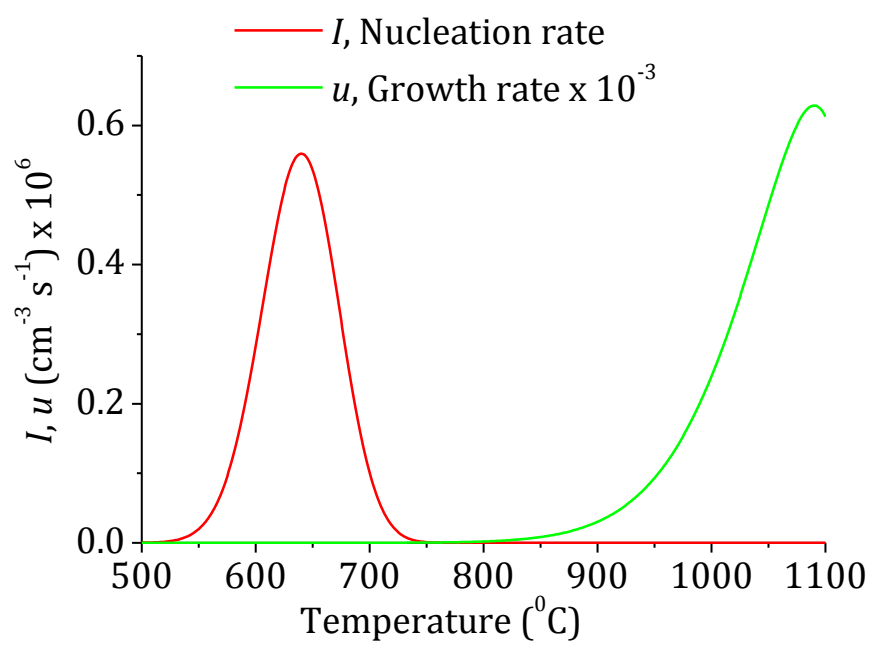

Fig. 1. Nucleation and growth rates for a hypothetical glass ceramic used in simulations.

An example of crystallization simulation is shown in Fig. 2, where the hypothetical material is undergoing 40 cycles of a triangular temperature ramp with low and high temperatures of 500 and $1100{ }^{\circ} \mathrm{C}$, respectively. The duration of one cycle is fixed at $100 \mathrm{~s}$, so that the temperature ramp is $\pm 12^{\circ} \mathrm{C} / \mathrm{s}$. The step-wise increase in $F_{V}$ is due to a stall in crystallization each time the cycle enters the low temperature stage. Note that nucleation is simulated as being continuous throughout the temperature-time trajectory, with a rate that depends on temperature only (as shown in Fig. 1). As can be seen from Fig. 2, the computed $F_{V}$ values overlap for the two methods, as a result of the mathematical identity of the two methods. This identity does not depend on the actual temperature-time trajectory, nor on the particular parameters values of nucleation and growth.

\footnotetext{
${ }^{\dagger}$ This is sensor element number 1 in the work of Fair et al. [8]
} 


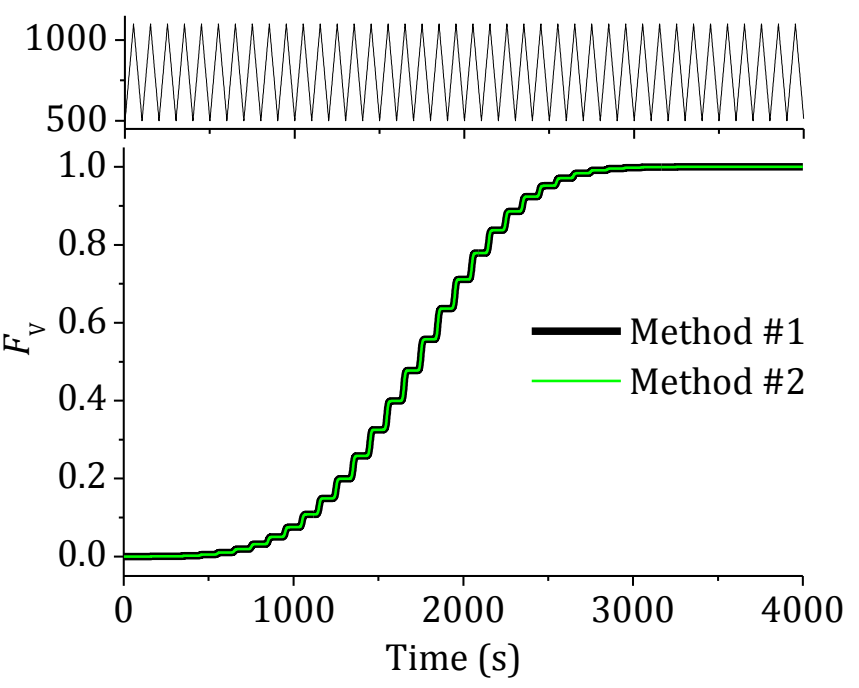

Fig. 2. Comparison of Methods \#1 (solid black line) and \#2 (solid green line), for a simulation of 40 triangular cycles between 500 and $1100{ }^{\circ} \mathrm{C}, \Delta t=1 \mathrm{~s}, \mathrm{~d} T / \mathrm{d} t=( \pm) 12$ ${ }^{\circ} \mathrm{C} / \mathrm{s}$. Note that the two methods are identical, regardless of the temperature-time trajectory and of the specifics of the nucleation and growth kinetics.

The computation time for an algorithm coded in C-language is shown for both Method \#1 and Method \#2 in Fig. 3, based on the use of a $3 \mathrm{GHz}$ processor and a memory of $4 \mathrm{~Gb}$. A comparison is made on the basis of varying the total number of time intervals. As can be seen, Method \#2 becomes approximately exponentially faster with number of time intervals. For instance, it takes approximately 2 hours to compute 10,000 intervals with M\#1, where this takes approximately 1 second with M\#2. It can be seen that Method \#2 still slows down with time, since the memory demands also increase. However, the number of computational steps is significantly reduced. For very large number of time intervals, intermediate storage on a hard drive might further speed up the computation in Method \#2, but this was not investigated in any detail.

Next, an analysis was made of the effect of time resolution $\Delta t$. For the same hypothetical material a triangular temperature ramp between 400 and $900{ }^{\circ} \mathrm{C}$ was chosen, with a period of 100 $\mathrm{s}$ and hence heating and cooling rates of $\pm 10^{\circ} \mathrm{C} / \mathrm{s}$. Fig. 4 shows the computed values $F_{V}$ as a function of time for values of $\Delta t=0.5,1,2,5$ and $10 \mathrm{~s}$. The triangular waveform parameters (high and low temperature and period) were chosen so as to exactly accommodate an integral number of time steps within each semi-cycle, thus avoiding sampling artifacts. As can be seen, as $\Delta t$ is decreased, the computed values of $F_{V}$ converge towards a curve for infinitesimally small $\Delta t$ (i.e., the continuous case) at values for $\Delta t$ of approximately $0.5 \mathrm{~s}$. Even for $\Delta t=1 \mathrm{~s}$, the systematical error is negligible, which is the main reason for choosing this interval in simulations mentioned in the previous sections. 


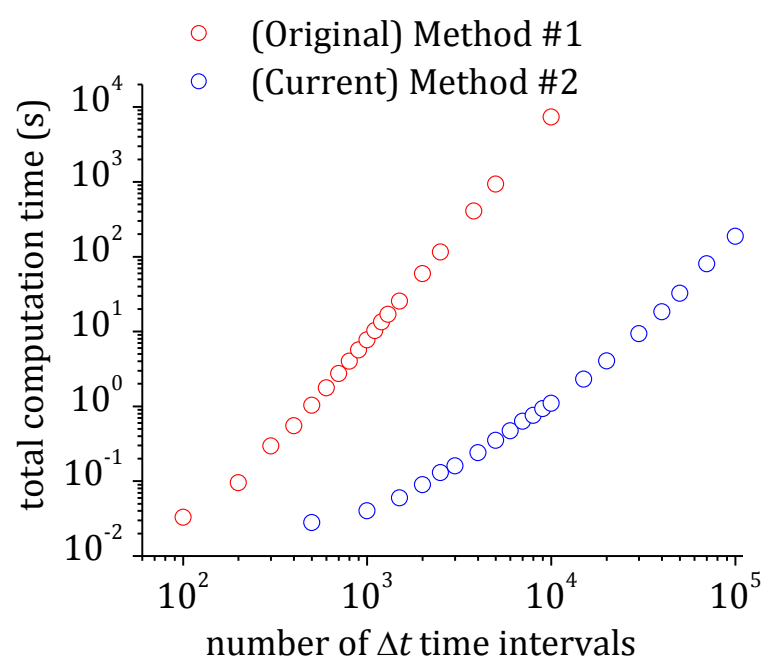

Fig. 3. Comparison of computation time as a function of number of time steps, for Methods \#1 and \#2. Method \#2 becomes exponentially faster the more time intervals are computed, as it does not rely on re-computation of the entire thermal history. Note that the difference in computation speed only depends on the number of time intervals, not on the specifics of the crystallization kinetics.

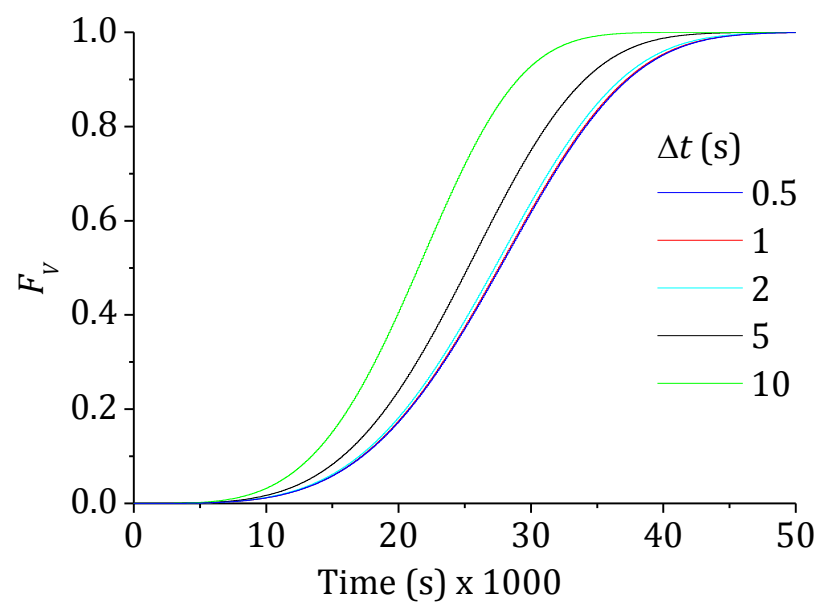

Fig. 4. Calculated fractional crystallization as a function of time for triangular temperature cycles between 400 and $900{ }^{\circ} \mathrm{C}$ with a period of $100 \mathrm{~s}$ and heating and cooling rates of $\pm 10{ }^{\circ} \mathrm{C} / \mathrm{s}$, for various values of the interval size $\Delta t$. For this case, a step size of 1 second or less is required for accurate simulation.

The minimal time resolution $\Delta t$ depends on the (average) heating and cooling rates. It was found from simulations that for an average $\mathrm{d} T / \mathrm{d} t= \pm 1{ }^{\circ} \mathrm{C} / \mathrm{s}, \Delta t$ should be $\sim 10 \mathrm{~s}$, whereas for $\mathrm{d} T / \mathrm{d} t=$ $\pm 100{ }^{\circ} \mathrm{C} / \mathrm{s}, \Delta t$ should be $\sim 0.1$, thus roughly scaling as $\Delta t_{\min } \sim 10 /(\mathrm{d} T / \mathrm{d} t)_{\text {ave }}$. 


\section{Discussion}

The modified Yinnon-Uhlmann algorithm presented here provides a means to compute fractional crystallinity with substantially higher computational speed than the original approach and with the same accuracy. The derivation was presented in the case of $m=3$ and is therefore relevant only for interface controlled growth of spherical grains. However, for different values of $m$ a similar derivation is possible, which is not shown here for brevity. Another simplification that was made is that of a negligible critical nucleus volume, which may lead to erroneous predictions in cases where the nucleation hold incubation time is long compared to the crystallization time or in the case where the fractional crystallization is still small, since the relative importance of $R_{i}^{*}$ increases in those cases. Nevertheless, it is relatively straightforward to include this parameter in the algorithm. Also, variations in nucleation and growth rates, for instance such as due to structural relaxation, may be present. Similarly, such effects can be included in a straightforward manner in the present algorithm by implementing variations in $u(T)$ and $I(T)$ with time. For such a modification, it would be important to know how structural relaxation or variances in $u(T)$ and $I(T)$ varies with $F_{V}$ so that the rate equations can be adjusted during the simulation. In summary, the method presented here is sufficiently flexible that different descriptions $u(T)$ and $I(T)$ may be used without lack of accuracy.

The main limitation of the present version of the algorithm, as of the original discrete version of the JMAK solution, is that it does not yet account for the effects of heating and cooling rates on the nucleation and growth rates. As shown by Shneidman and Uhlmann [4,5], the quasi-steadystate (QSS) approximation may break down under even modest heating and/or cooling rates as low as $0.01 \mathrm{~K} / \mathrm{s}$. In the same work, however, it is shown that the departure from the steady-state condition can be computed, by means of a time-dependent nucleation frequency expressed with correction factor $\phi(n)$ to the steady state nucleation frequency $I_{s t}$, as

$$
I \sim I_{s t} \phi(n)
$$

where $n$, the "nonstationary index", is defined as

$$
n=-\tau \frac{\partial}{\partial t} \frac{W_{*}}{k T}
$$

where $W_{*}$ is the nucleation energy barrier (i.e., the minimal work required to form a cluster of monomers with critical size), $k T$ is the usual thermal energy, and $\tau$ is a relaxation parameter, which is defined in terms of the crystallite size dependent growth rate, $U(r)$, at the critical radius $r_{*}$ :

$$
\tau^{-1}=\left(\frac{d U}{d r}\right)_{r=r_{*}}
$$


Under steady state conditions, the correction factor $\phi(n)$ approaches 1 as $n$ approaches 0 . Since $W_{*}$ depends on temperature, the factor $\frac{\partial}{\partial t} \frac{W_{*}}{k T}$ in effect accounts for the cooling or heating rate, as is the definition of $\tau$. Then, to a first approximation, the correction factor $\phi(n)$ can be implemented in the present algorithm (or a similar algorithm based on the standard JMAK model) for the general case of arbitrary thermal history (i.e., including variable heating and cooling rates).

The non-QSS effects in crystallization have been analyzed in detail by Kelton, which resulted in a complete numerical solution of the Becker-Döring system of equations for describing the cluster dynamics [3]. By implementing the non-QSS correction factor in the present algorithm, the different methods for computing the crystallized volume fractions ought to provide converging values.

Finally, it was shown by Shneidman an Uhlmann that the double integral can also be rewritten as a system of 4 ordinary differential equations for the moments of the distribution of nuclei over sizes [5], which may provide a faster means for computing the fractional crystallinity. A full comparison of the different methods, although of much interest, is beyond the scope of the present paper. Therefore, it is of interest to compare the different approaches in terms of speed and accuracy in further work.

\section{Conclusions}

A fast algorithm is presented for computing the classic JMAK double integral formulation under non-isothermal conditions within the quasi-steady-state (QSS) approximation. The increase in computation speed over the original discrete description by Yinnon and Uhlmann from which it was derived, is the result of not having to re-compute the entire thermal history. The improved algorithm is shown to be identical in accuracy to the original approach. Furthermore, possible extensions to the non-QSS domain are discussed, which enable a more general algorithm for fast computation of non-isothermal crystallization processes under arbitrary heating and cooling rates.

\section{Acknowledgements}

Part of this work was funded by the U.S. Air Force SBIR program (contract FA8650-08-C-5204 to MetroLaser). 


\section{References}

1 H.R. Yinnon and D.R. Uhlmann, J. Non-Cryst. Sol. 54 (1983) 253.

2 D.R. Uhlmann and H.R. Yinnon, in Glass: Science and Technology, Vol. 1- GlassForming Systems, Academic Press, New York (1983) 1-47.

3 K.F. Kelton, J. Non-Cryst. Sol. 163 (1993) 283.

4 V.A. Shneidman and D.R. Uhlmann, J. Chem. Phys. 108 (1998) 1094.

5 V.A. Shneidman and D.R. Uhlmann, J. Chem. Phys. 109 (1998) 186.

6 R.W. Hopper, G. Scherer and D.R. Uhlmann, J. Non-Cryst. Sol. 15 (1974) 45.

7 M.J. Davis and P.D. Ihinger, J. Geophys. Res. - Sol. Earth 107 B11 (2002) 2284.

8 G.E. Fair, R.J. Kerans and T.A. Parthasarathy, Sensors and Actuators A 141 (2008) 245. 